\title{
Incentive for the Research that Highlights the Relationship between Smoking and the Development of Chronic Obstructive Pulmonary Disease (COPD)
}

\section{Moreira Santos TM* and Vesentini G}

Faculty of Medicine of Botucatu, São Paulo State University-UNESP, Brazil

*Corresponding author: Moreira Santos TM, Faculty of Medicine of Botucatu, São Paulo State University-UNESP, Brazil, Tel: +55 14 991043232; Email: thymy25@hotmail.com

Rec date: May 09, 2017; Acc date: June 08, 2017; Pub date: June 15, 2017

Copyright: (C) 2017 Moreira Santos TM, et al. This is an open-access article distributed under the terms of the Creative Commons Attribution License, which permits unrestricted use, distribution, and reproduction in any medium, provided the original author and source are credited.

Citation: Moreira Santos TM, Vesentini G (2017) Incentive for the research that highlights the relationship between smoking and the development of chronic obstructive pulmonary disease (COPD). Chron Obstruct Pulmon Dis 2: 21.

\section{Letter to Editor}

Smoking, one of the most important public health problems, is responsible for a large number of preventive deaths in the world. It is also the main risk factor for the development of numerous diseases. For the last few decades, it has become a relevant topic for studies, especially because of the damage to the population health with negative consequences to the social, economic and sanitary sector.

Cigarette users are exposed to a variety of chemical agents that can cause a series of detrimental health effects. Previous studies have shown that smoking can cause irreparable damage to the genetic material, cell damage and, consequently, the development of pulmonary diseases such as COPD.

Likewise, research showing the relationship between smoking and the development of lung diseases should be further explored in order to understand the damage caused by smoking and to encourage people to quit smoking.

Although there are several studies about this specific topic, there are few studies that investigate the chronic asymptomatic smokers and its relation with mild and moderate COPD. Such research theme would be essential to demonstrate the changes caused by the use of cigarrete and how these changes are close to patients who already have the disease.

Our research team investigates the changes caused by cigarette and studies the relationship between chronic asymptomatic smoker and non-smokers, and patients with mild and moderate COPD. Results are being found (data not yet published), which could enlighten the pathophysiological process involved between cigarette and the risk of disease, including the risk of autoimmune diseases. Among our study variables, we seek to clarify the action of non-physiological stress caused by cigarettes, the genotoxicity, the inflammatory process and the immunological changes.

We believe that research in this area tends to improve the understanding, both in basic science and in clinical issues of the pathological processes related to the use of cigarette, which may lead to the development of new therapies. Molecular and immunological techniques may be essential tools for detecting specific molecules and solving existing issues involving these diseases.

These studies may open pathways for new research and contribute to the advancement of knowledge about the risk factor involved in smoking and COPD. We hope that this letter will encourage fellow scientists in the areas to invest in this field of research whose main objective is to clarify the changes caused by cigarette and to show that these changes are close and connected to the pathophysiology of COPD. Also, this research can be used as an incentive to smoking cessation and as strategies to effect reduction, consequently decreasing COPD in the population. 